Francesco Arcidiacono ${ }^{1}$, Alexandre Bezençon, Esther González-Martínez HEP-BEJUNE \& Université de Lausanne (Suisse)

\title{
Article scientifique
}

\section{Séances entre enseignants de classes inclusives: un espace de déploiement de la réflexivité}

Résumé: Cette étude porte sur des séances de travail entre un enseignant spécialisé et un enseignant généraliste qui collaborent dans le cadre d'une classe inclusive. Les enseignants nous ont procuré des enregistrements audio de leurs séances et accepté de participer à des entretiens individuels sur celles-ci. L'analyse conversationnelle de la séance montre que les enseignants préparent des activités éducatives en justifiant leurs propositions. Ils confrontent leurs connaissances et expériences ainsi que leurs évaluations des compétences des élèves et de la difficulté des tâches. En échangeant, ils tissent un espace de déploiement de la réflexivité dans et sur l'action professionnelle. Lanalyse de contenu de lentretien confirme ces résultats et permet d'expliciter des réponses des enseignants mis au défi de la classe inclusive. Ces éléments peuvent contribuer à une planification efficace des échanges entre acteurs éducatifs dans le but de dépasser les évaluations qui sur-ou sous-estiment leurs compétences et permettre un alignement réciproque en classe inclusive.

Mots-clés: Enseignant, classe inclusive, séance de travail, réflexivité.

\section{Introduction}

Les enseignants d'écoles hétérogènes en Suisse accueillent des élèves en difficulté d’apprentissage. Ils sont responsables de classes à visée inclusive cherchant à favoriser lépanouissement des élèves, les processus d'autonomisation, l'acquisition des connaissances, louverture à autrui et la participation sociale (CDIP, 2007). Cet article présente une étude sur la réflexivité lors de séances de travail entre enseignants de classes inclusives. Parmi les mul- tiples significations du mot réflexivité (Lynch, 2000), nous avons retenu celle d'une capacité interprétative consistant à considérer des actions éducatives et à en rendre compte (Vacher, 2011). Inspirés par des approches socioculturelles et conversationnelles, nous envisageons cette capacité dans une perspective praxéologique. Nous nous écartons donc de visions internalistes, individualisantes et isolationnistes de la capacité réflexive pour nous intéresser à sa nature située, plurielle, construite en interaction ainsi qu’à son caractère dynamique, émergeant au fil

1 Francesco.Arcidiacono@hep-bejune.ch

Copyright $\odot 2017$ by the authors, licensee Teacher Education Faculty University of Belgrade, SERBIA.

This is an open access article distributed under the terms of the Creative Commons Attribution License (CC BY 4.0) (https://creativecommons.org/licenses/by/4.0/), which permits unrestricted use, distribution, and reproduction in any medium, provided the original paper is accurately cited. 
des échanges entre les interactants (Mondada \& Pekarek Döhler, 2000, 2006).

Notre intérêt pour les interactions de travail entre enseignants de classes inclusives découle d'une volonté de mieux comprendre l'équilibre entre progression et stagnation dans les apprentissages en contexte scolaire hétérogène. Nous avons considéré important de saisir les dynamiques à l’œuvre entre enseignants spécialisés et enseignants généralistes : envisager les difficultés des élèves à partir d'une double perspective amène en effet à articuler des positionnements divergents. Lors de séances de travail, les enseignants avancent des propositions sur des activités éducatives, les confrontent et les justifient. Nous soutenons que ce travail nourrit la construction d'une réflexivité dans et sur l'action professionnelle (Vacher, 2011).

Notre étude est basée sur l'analyse conversationnelle d'enregistrements audio de séances entre les enseignants d'une classe inclusive, complétés par des entretiens individuels avec ces mêmes enseignants. Dans la suite de l'article, nous exposons le cadre théorique de notre étude, sa méthodologie ainsi que nos principaux résultats. La conclusion synthétise les fonctions de la séance de travail et son importance pour le déploiement de la réflexivité des enseignants au sujet de leurs actions.

\section{Cadre théorique}

Notre cadre théorique se nourrit de différentes démarches fournissant des clés pour articuler les interactions entre enseignants, la mise en place des activités éducatives, leur justification et la réflexivité à l'œuvre lors des échanges.

Dans la perspective ethnométhodologique, la réflexivité est une propriété inhérente à toute action sociale (Czyzewski, 1994 ; Lynch, 2000). Il ne s'agit pas d'une capacité des individus, mais de la propriété de leurs actions à devenir intelligibles (accountable) en cours de réalisation, de manière parfaitement ordinaire et inéluctable. Une approche conver- sationnelle (Sacks, Schegloff \& Jefferson, 1974) permet toutefois de spécifier praxéologiquement la réflexivité en tant que capacité des individus. Elle s'intéresse à la dimension socialement construite des entités cognitives, produites en situation, au fil des échanges entre les participants à des cours d'action. Dans ce sens, la réflexivité est située, partagée, plurielle et dynamique parce que façonnée moment par moment (Mondada \& Pekarek Döhler, 2000, 2006). Cette approche conversationnelle nous invite à considérer la possibilité dêtre réflexif comme une activité à laquelle les individus peuvent se consacrer tout particulièrement lors de certaines interactions, dont il est possible de retracer les actions constitutives et les pratiques langagières qui permettent de les accomplir.

Dans une perspective socioculturelle, les interactions de la vie quotidienne constituent une force prégnante dans le développement et la socialisation des individus (Pontecorvo \& Arcidiacono, 2007) : les processus cognitifs sont façonnés lors d'interactions sociales où ils deviennent observables. Par ailleurs, les échanges langagiers constituent un espace potentiel de partage dans lequel les participants produisent et négocient ensemble des significations. Ainsi, différentes études ont montré que les activités quotidiennes dans les contextes éducatifs sont des moments privilégiés lors desquels adultes et enfants mettent en forme et donnent sens à leur environnement par des conversations ordinaires (Arcidiacono, 2013 ; Pontecorvo \& Arcidiacono, 2014). Ces échanges ont souvent une dimension argumentative puisqu'ils conduisent à une remise en question de la validité des idées en les confrontant à l'examen d'autrui. Notre étude s'inspire de cette perspective socioculturelle qui articule la dimension socialisante du langage au déploiement de capacités cognitives et à la construction argumentative de significations (Krummheuer, 2000 ; Arcidiacono, 2015).

Nous inscrivons également notre étude dans le sillage des travaux qui se penchent sur la fonction sociale et relationnelle du langage en milieu scolaire. 
A défaut de considérer la relation privilégiée entre l'enseignant et ses élèves, nous nous intéressons aux interactions verbales entre enseignants lors desquelles ceux-ci élaborent des activités éducatives et les justifient. Les travaux de Roger, Ruelland et Clot (2007) nous permettent de considérer le métier d'enseignant de manière pluridimensionnelle, en sorientant autour de trois pôles : le premier concerne la transmission de lobjet du savoir ainsi que son appropriation par les élèves. Il permet d'interroger le rapport de soi à lautre autour de l'objet à enseigner et de réfléchir sur les conduites à faire adopter aux élèves. Le deuxième pôle considère la relation que les élèves créent par l'activité sur un même objet. Il est ici question du rapport aux camarades, mais aussi à d'autres enseignants, parents ou intervenants $\mathrm{du}$ système socio-éducatif. Le troisième pôle s'exprime dans le rapport de l'enseignant ou de lélève aux savoirs scolaires et pédagogiques socialement situés. Cette articulation rappelle le triangle didactique suggéré par Houssaye (1992) dans lequel l’enseignant, l'apprenant et le contenu de l'enseignement sont considérés comme trois entités liées par un rapport de constitution réciproque. De plus, la dynamique relationnelle qui lie les différents éléments est pensée non pas comme un produit donné, mais plutôt comme une émergence (Dos Santos Mamed, 2014). En ce sens, agir au sein du triangle didactique revient à s'engager dans un cours d'action dont les contours sont négociés à chaque instant, dans lequel les participants peuvent s'aligner et réajuster leurs actions en fonction de la mise en tension des trois pôles. Cette perspective, en termes de cours d'action conjoints, offre la possibilité de saisir les trois pôles du triangle didactique en tant qu'entités réalisées par les participants lors de leurs échanges.

Notre étude s'inspire également du concept de réflexion sur l'action que Schön (1994) avait défini comme "le retour analytique sur une interaction passée" (p. 19). Lors de leurs échanges, les enseignants examinent la meilleure manière de transmettre l'objet du savoir à leurs élèves. Vacher (2011) considère ces discussions entre pairs comme une composante du "réfléchir le faire" (p. 72). Ils permettent aux interlocuteurs de développer un discours propre en intégrant souvent d'autres discours, réels ou imaginés, de participants en présence ou de tiers absents (Grossen \& Salazar Orvig, 2011).

Pour résumer, notre étude de la production de la réflexivité lors déchanges entre enseignants est inspirée de démarches qui considèrent la réflexivité dans une perspective praxéologique. Notre cadre théorique nous invite à rendre compte de la manière dont les enseignants élaborent des activités éducatives et reconnaissent leurs actions comme sorientant dans ce but. Il nous fournit également des clés pour saisir le travail de justification du bien-fondé des activités que les enseignants effectuent lors d'échanges avec leurs collègues.

\section{Méthodologie}

\section{Participants}

Cet article se base sur une étude de cas et explore la capacité réflexive qui émerge lors de séances de travail entre un enseignant spécialisé et un enseignant généraliste autour d'activités scolaires d'une classe inclusive. Les participants sont deux enseignants d'une classe de $5^{\text {ème }}$ année $\mathrm{HarmoS}^{2}$ dans un établissement de l'ouest vaudois (Suisse). Ils y travaillent en dyade depuis une année. Claudia (nom fictif), âgée de 60 ans, est détentrice d'une Licence en Psychologie et Sciences de l'Education et a une expérience de plus de 40 ans dans le milieu de l'enseignement (plus particulièrement dans l'enseignement spécialisé). Dans ce domaine, elle détient un titre de Master décerné par une Haute Ecole Pédagogique en 2009. Jef (nom fictif), âgé de 30 ans, a suivi une formation pédagogique en enseignement primaire. Il détient dans ce domaine un titre de Bachelor décerné par une Haute Ecole Pédagogique et enseigne depuis une année.

2 Accord intercantonal sur l'harmonisation de la scolarité obligatoire. 
Les deux enseignants ont accepté de participer à létude de manière volontaire et sans contrepartie financière. Ils ont répondu à un appel de l'équipe de recherche pour une étude présentée en des termes très généraux comme étant axée sur les pratiques éducatives en classe inclusive.

\section{Contexte: classe et établissement scolaire}

Claudia s'occupe d'un groupe de 12 élèves en difficulté d'apprentissage. Elle est responsable et enseignante pédagogique de la classe. Le groupe est partagé en deux classes inclusives : une de $5^{\text {ème }}$ année HarmoS (dont Jef est l’enseignant généraliste) et une de $6^{\text {ème }}$. Le co-enseignement, ainsi que la collaboration entre enfants avec et sans difficultés, vise l'épanouissement, l'autonomisation, l'acquisition des connaissances et louverture à autrui par des processus de socialisation. Les enseignants se donnent pour objectif l'évolution des enfants en difficulté au sein de classes ordinaires. La classe est donc un espace interactif hétérogène où s'entrecroisent des discours, des rôles et des actions très diverses. Cette hétérogénéité est reconnue au niveau de lorganisation de la classe de manière à faciliter les apprentissages. Si une difficulté se présente, l'enseignante spécialisée peut par exemple se retirer dans sa classe pour étayer des notions mal intégrées et ensuite revenir dans la classe ordinaire. Létablissement scolaire luimême considère les classes inclusives comme un modèle à suivre pour l'apprentissage et le développement affectif, cognitif et social des élèves.

\section{Données : collecte et traitement}

Les données ont été récoltées durant l'année scolaire 2015-2016. Il s'agit d’enregistrements audio de séances de travail entre enseignants (c'est-à-dire des situations naturelles d'action non provoquées par les chercheurs), ainsi que dentretiens semi-directifs. Les premiers nous renseignent sur « la réflexivité dans l'action ", les deuxièmes le font au sujet de « la réflexivité sur l’action" (Vacher, 2011).
Les enregistrements audio des séances de travail entre les enseignants ont eu lieu après les cours. En effet, les deux enseignants avaient l'habitude de se rencontrer après avoir enseigné, dans le but de faire le point sur lorganisation des tâches ainsi que les éventuelles difficultés rencontrées lors de la séance. Ces entrevues procurent du feedback et permettent la planification des activités à venir. Nous disposons d'enregistrements de trois séances de ce type. Pour cet article, nous avons retenu plus particulièrement la troisième séance. L'enregistrement a été réalisé sans la présence du chercheur, Jef sétant chargé d'activer et d'éteindre l'appareil. L'échange entre les deux enseignants a eu lieu dans la salle de classe pour une durée de 70 minutes.

Le traitement de l'enregistrement de la séance ainsi que son analyse suivent l'approche de l'analyse de conversation (Sacks, Schegloff \& Jefferson, 1974). Nous avons commencé par transcrire l'enregistrement dans son intégralité selon les conventions propres à l'approche (cf. annexe). L'analyse consiste à identifier ce que les interlocuteurs font en échangeant et la manière de l'accomplir. La réflexivité qui caractérise le travail réalisé lors des séances est ainsi saisie en cours de production, à partir des actions des interlocuteurs (Radišić \& Baucal, 2016). Notre analyse se concentre sur quelques extraits de la séance des deux enseignants qui sont particulièrement pertinents par rapport à l'objet d'étude. La sélection des extraits a été faite en deux étapes. Tout d'abord, le premier et le deuxième auteur ont opéré un choix, travaillant de manière indépendante. Ensuite, ils ont examiné ensemble leurs sélections respectives, qui étaient à $80 \%$ concordantes, et discuté du choix défini jusquà parvenir à un accord. Suivant un principe propre à l'analyse de conversation, les chercheurs ont jugé de la pertinence des extraits par rapport à la thématique de l'étude en suivant les orientations des participants : c'est-à-dire qu'ils ont retenu des passages où les interlocuteurs eux-mêmes montraient que léchange était en rapport avec une activité réflexive à propos des activités éducatives (Arcidiacono, Baucal \& Buđevac, 2011). 
Le deuxième type de données consiste en un entretien semi-directif réalisé par le deuxième auteur individuellement avec l'enseignante spécialisée. Le but était de revenir sur des notions ayant émergé lors des séances enregistrées et de demander de les expliciter. Les catégories ont émergé en effet des autres séances enregistrées (cf. théorie enracinée). Ces entretiens ont confronté les enseignants à leurs pratiques, à la manière de mettre en place des activités éducatives et de les justifier. Le chercheur a élaboré un guide d'entretien à la suite d'une première analyse synoptique des enregistrements des séances entre enseignants. Ce procédé a permis d'identifier quatre grandes questions sur lesquelles le guide d'entretien s'est concentré : l'approche expérientielle; léquilibre ; l'évaluation ; la temporalité. D'autres questions en lien avec l'activité enseignante ont émergé lors des entretiens, telles que le travail en dyade et l'équilibre des tâches. Elles ont également été prises en considération lors de l'analyse. Ces entretiens ont engagé les enseignants dans un processus métacognitif, interrogeant la cohérence de la pratique éducative et parfois son pendant incohérent. La confrontation des enseignants avec des éléments tirés des enregistrements de leurs séances constitue un moment de retour analytique et de réflexion sur l'action, marqué par la mise à distance et le regard critique envers sa propre pratique. Les entretiens ont été transcrits et analysés selon une approche thématique de contenu (Coffey \& Atkinson, 1996). Dans le présent article, l'analyse se concentrera sur l'entretien avec Claudia, l'enseignante spécialisée, dont la durée est de 25 minutes.

L'ensemble des données - séances entre enseignants et entretiens individuels avec le chercheur - donnent à voir l'investissement des enseignants pour traiter des obstacles sous-jacents à leurs activités éducatives et plus particulièrement à l'inclusion des enfants en difficulté d’apprentissage.

\section{Analyse des données et résultats}

Pour commencer, nous présenterons notre analyse conversationnelle de la séance entre enseignants, qui rend compte de la production de la réflexivité en situation. Ensuite, l'analyse de l'entretien avec l'éducatrice spécialisée nous permettra d'exposer sa réflexion a posteriori au sujet de sa propre pratique. Dans la suite du texte, les nombres entre parenthèses correspondent aux numéros de ligne des extraits ci-dessous. Tous les prénoms ont été remplacés par des pseudonymes.

\section{Analyse de la séance entre enseignants}

L'échange entre enseignants : projeter et justifier

L'extrait 1 présente un échange entre Claudia, l'enseignante spécialisée, et Jef, l'enseignant généraliste, autour de l'activité cyber-défi (www.cyberdefi.ch). Il s'agit d'un concours qui a pour but de développer l'esprit scientifique et la recherche d'informations. Les élèves réalisent des épreuves en ligne et reçoivent immédiatement leurs résultats par le même moyen. Au cours de l'échange, Jef et Claudia discutent de la préparation de la classe en vue de la participation à ce concours. De ce fait, léchange a une forte dimension projective. Les enseignants construisent ensemble, à haute voix, une réflexion commune sur la manière de mener à bien l'activité de préparation.

Au début de l'extrait, Claudia s'exprime au sujet de la préparation de la classe en se mettant à la place de Jef (c'que tu peux faire c'que tu pourrais faire, ligne 1). Avant de faire sa proposition, elle la justifie (puisqu'cest votre premier et pi que vous l'avez jamais fait, lignes 1-2). Ensuite, elle se sert du discours direct pour représenter Jef en train de s'adresser à sa classe avec les mots quelle lui propose de produire (oké on va faire quelque chose d'un peu particulier, lignes 2-3). Jef lui indique le moment dans le programme où il pourrait introduire l'activité quelle accepte tout en signalant qu'il n'est pas indispensable d'entraîner les enfants à l'activité. Aux lignes 8-9, 


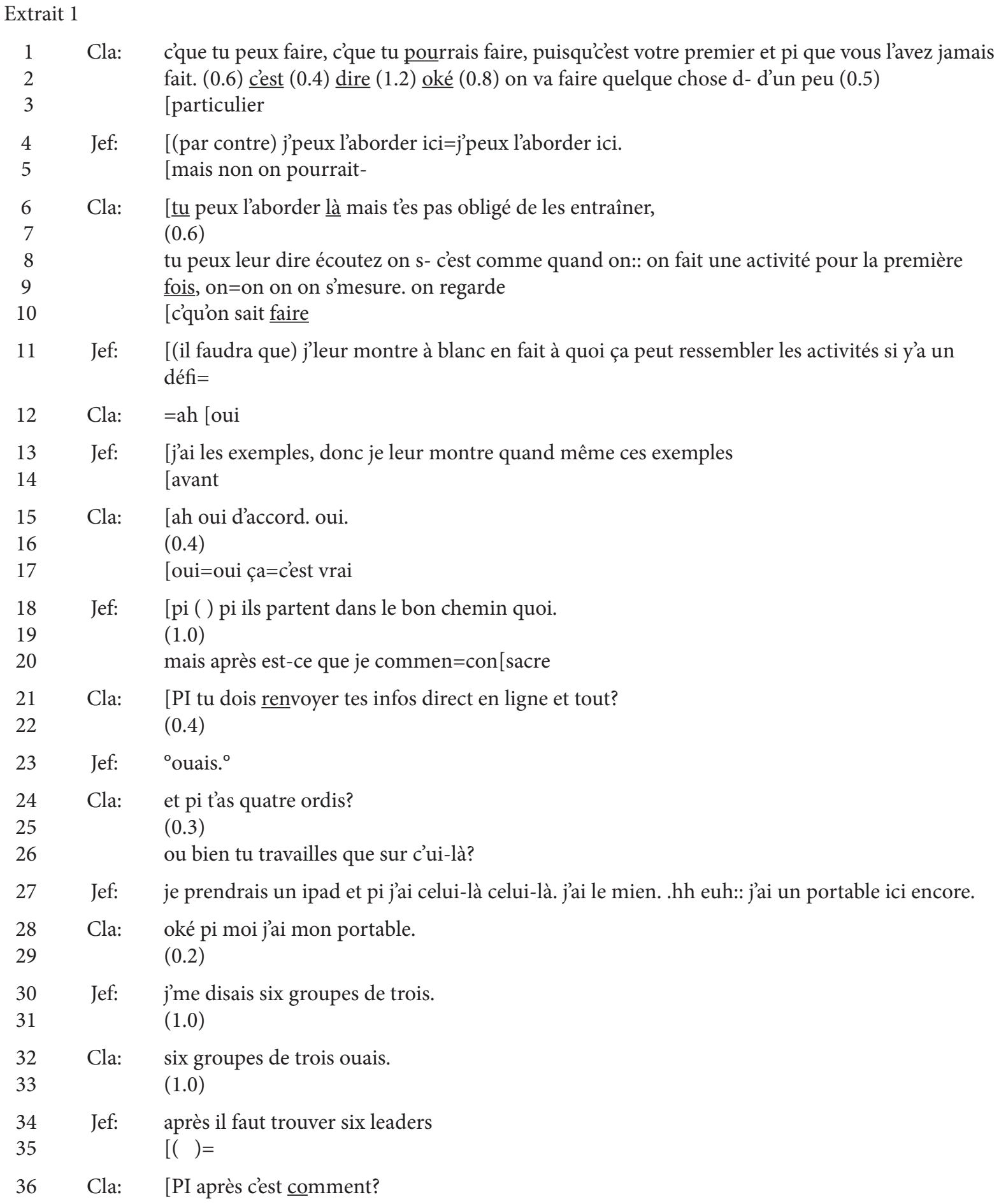




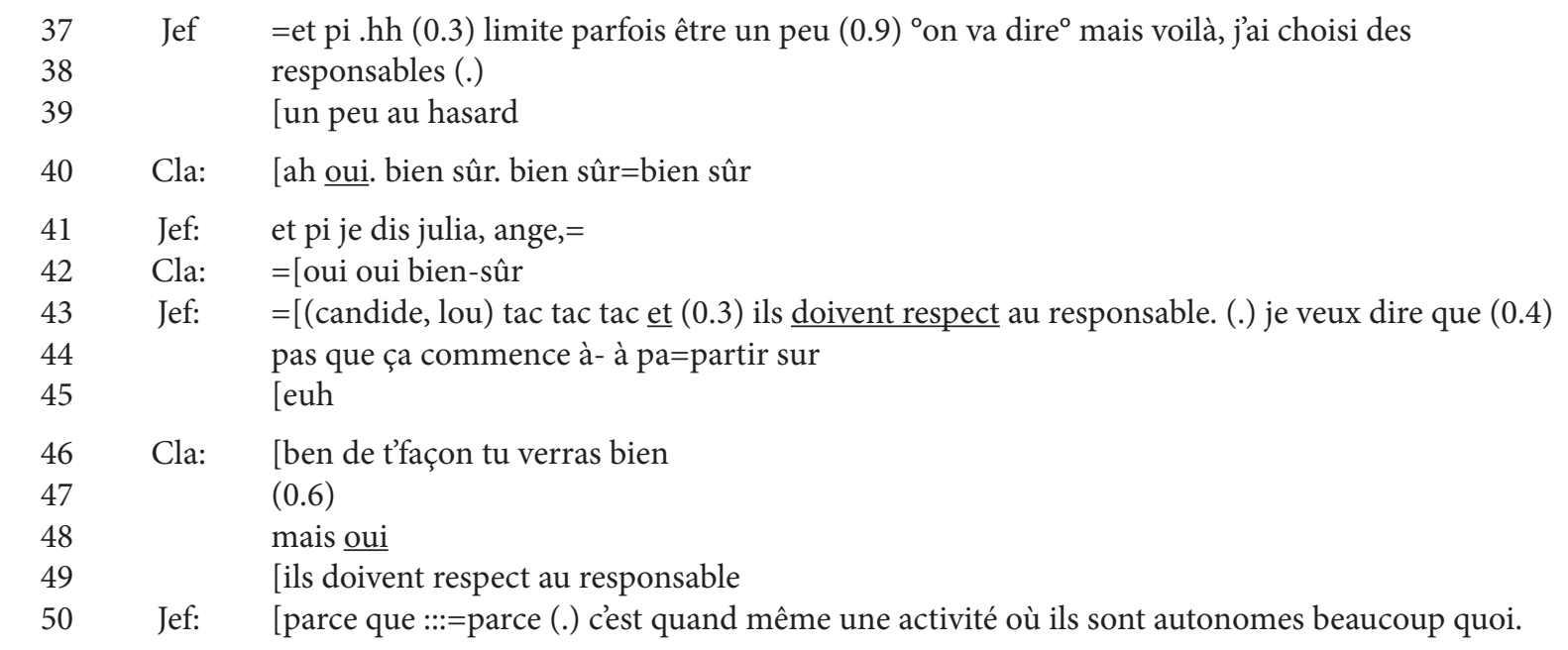

Claudia continue de s'exprimer comme si elle était l’enseignant généraliste sadressant à la classe (tu peux leur dire, ligne 8). Cette proposition, basée sur la capacité de décentration de Claudia, simule une interaction qui na pas encore eu lieu dans le but de préparer l'activité à mener en classe. Elle propose d'amener le cyber-défi aux élèves, en se basant sur le rappel utilisé lors de l'introduction de nouvelles tâches (écoutez on s c'est comme quand on euh on fait une activité pour la première fois on on s'mesure on regarde c'qu'on sait faire, lignes 8-9). Jef reste toutefois orienté envers la manière de préparer concrètement les enfants pour le concours. Il propose une alternative à entraîner les enfants, ce qui avait été écarté par Claudia aux ligne 6. Elle consisterait à leur montrer des exemples des épreuves (lignes 11-14). La proposition est justifiée comme permettant aux élèves de commencer l'épreuve du bon pied (ligne 18). Alors que Jef fait référence à la suite de la préparation des élèves (ligne 20), Claudia change de topic pour le questionner au sujet de lorganisation pratique du concours en termes de besoins informatiques. Les enseignants passent en revue les appareils à disposition, qui sont au nombre de six (lignes 24-28). Ceci correspond avec la proposition avancée ensuite par Jef, et ratifiée par Claudia, de distribuer les élèves en six groupes. Jef sengage ensuite sur la question de lorganisation interne des groupes, même si Clau- dia semble lui demander de décrire le déroulement de l'épreuve (ligne 36). Tout d'abord, Jef est orienté vers la justification de la désignation par l'enseignant des leaders des groupes. Dans un nouveau mouvement projectif, il se représente en train de s'adresser à ce sujet à la classe. Claudia est entièrement d'accord avec la proposition et Jef poursuit avec la présentation pour les élèves de lobligation de respecter les responsables de groupe. Ensuite, Jef semble faire référence à la situation que cette mesure cherche à éviter (pas que ça commence à partir, lignes 43-44), mais Claudia ne présuppose pas qu'elle soit efficace (ben de t'façon tu verras bien, lignes 46-47). Le caractère non-préférentiel de cette réponse engage Jef dans un processus de justification de sa proposition (parce que parce c'est quand même une activité où ils sont autonomes beaucoup quoi, ligne 50).

Lextrait montre la dimension projective de léchange entre enseignants lors duquel il s'agit d'organiser l'activité cyber-défi à conduire en classe. Lenseignante spécialisée se met à la place de l'enseignant généraliste et lui propose une manière de préparer les élèves à l'activité. Elle représente Jef en train de s'adresser à la classe et énonce les mots qu'elle lui propose d'utiliser. Quant à Jef, il reste centré sur ses propres actions de préparation de la classe et d'organisation des groupes. Il avance des propositions et répond aux questions de Claudia. Les deux 
enseignants justifient leurs propositions en avançant des éléments qui montrent leur bien-fondé en termes pédagogiques. Une désaffiliation intervient aux lignes 48-49, lorsque Claudia met en doute l'efficacité de l'obligation de respect aux responsables, ce qui déclenche une nouvelle justification de la part de Jef. Tout au long de l'extrait, les enseignants préparent l'activité, mais font aussi preuve de réflexion par rapport à leurs choix.

\section{Réflexivité et différentiel de connaissances}

L'équilibre à trouver dans les classes, entre un mouvement vers le progrès des élèves et la prise en compte de leurs difficultés, est un souci que les enseignants expriment souvent lors de leurs échanges. La nature hétérogène de la classe - au niveau des élèves, mais aussi des enseignants - sollicite des discussions lors desquelles les enseignants cherchent des compromis et rendent visibles leurs connaissances ainsi que leurs rôles dans le cadre scolaire. Dans l'extrait 2, léchange porte sur la possibilité d'introduire la fiche «Ecureuil » que Claudia, l’enseignante spécialisée, considère difficile.

L'extrait débute avec la proposition de Jef d'introduire la fiche "Ecureuil " (lignes 1-2). Claudia la qualifie de difficile laissant ainsi entendre qu'elle ne conviendra pas aux élèves. Cette séquence met à nouveau en évidence le travail de préparation des activités éducatives et de justification des choix que nous avons déjà observé dans l'extrait 1 . Ensuite, Jef demande confirmation de lévaluation de la fiche, en la remettant en question avec le mot vraiment (c'est vraiment difficile?, ligne 4). Claudia réplique en indiquant un sous-groupe délèves qui pourrait bien sen sortir (ben pas pour Julia et les autres, ligne 5) mais réaffirme son évaluation puis la justifie en indiquant le travail que la fiche demande aux enfants de réaliser (mais oui parc'qu'il faut compter faut sauter, ligne 5). La réplique montre la catégorisation que l'enseignante spécialisée fait de ses élèves : Julia et les

Extrait 2

1 Jef: mais on a quà (0.2) écureuil=jaimerais bien voir parce que:

$2 \quad$ [après

3 Cla: [ECUreuil c'est une fiche difficile.

4 Jef: $\quad$ c'est vraiment difficile?

5 Cla: uhm:: ben pas pour Julia et les autres, mais oui parc'qu'il faut compter, faut sauter:

$6 \quad(0.7)$

$7 \quad$ oui. (.) c'est la fiche dix-huit.

8 Jef: oui mais là c'est d'nouveau c't'histoire de: justement des rythmes, (0.6) euh::

9 Cla: OUais c'est pour ça qu'i

10 [faut pas

11 Jef: [faut pas=faut pas freiner les autres quoi

12 [le but c'est justement (pour pouvoir organiser pour que-)

13 Cla: [non=non- (0.3) non alors c'est pour ça.

$14 \quad$ jallais dire et peut-être qu’on peut re:noncer à certaines choses avec les autres

$15 \quad(0.9)$

16 et pi la fiche écureuil (0.7) dix-huit voilà. (0.5) parc'quen fait si tu veux, (0.2) tu te déplaces $(0.3)$

17 donc cinq plus six. on:ze (.) tu dois rejoindre le cent-cinquante qui est là. 
autres servent à designer implicitement les plus performants. A son tour, Jef produit une contre-assertion (format $\mathrm{X}$ mais $\mathrm{Y}$ ) qui problématise la réponse de Claudia. Il concède que la fiche puisse présenter des difficultés pour certains élèves mais met en avant l'importance de ne pas freiner les autres (ligne 11). Cet échange manifeste les équilibres à trouver en classe inclusive auxquels Jef fait référence avec l'expression là c'est d'nouveau c't'histoire de justement des rythmes (ligne 8). L'extrait met aussi en évidence les rôles de l'enseignante spécialisée qui représente les élèves en difficulté et de l'enseignant généraliste qui représente les élèves de la classe ordinaire. Claudia explicite le sens de ces interventions (jallais dire, ligne 14) et propose un compromis consistant à renoncer à faire certaines choses avec une partie des élèves (lignes 15-16). Elle s'engage ensuite dans la présentation de la fiche en indiquant encore une fois ce qu'elle demande aux élèves de réaliser.

Nous observons que l'extrait met en avant un différentiel de connaissances entre Jef, l'enseignant novice, et Claudia, l'enseignante expérimentée. Le premier souhaite introduire la fiche, la deuxième la connaît, donne son numéro (ligne 7 ) et présente à Jef le travail qu'elle implique pour les élèves (ligne 5 et 16-17). Manifester cette connaissance est utile à Claudia pour justifier son évaluation de la fiche qui inviterait à ne pas l'introduire sans autre pour l'ensemble de la classe. Cet extrait montre encore une fois que la séance sert à préparer les activités éducatives et à justifier les choix des enseignants. Il met aussi en évidence le défi de l'équilibre propre aux classes inclusives et les positions que les participants prennent par rapport à ce défi en fonction, respectivement, de leurs rôles d'enseignante spécialisée et enseignant généraliste. Il montre enfin l'utilité de la séance pour confronter les propositions d'un enseignant aux connaissances de l'autre, et développer ensemble une capacité réflexive sur les actions à entreprendre.

\section{Expérience pratique et réflexivité partagée}

Nous avons vu que l'évaluation différenciée en fonction du rôle de l'enseignant - enseignant spécialisé ou généraliste - constitue un trait de l'échange. Les enseignants doivent examiner la manière de faire en tenant compte des défis des classes inclusives. Dans l'extrait 3, Claudia propose d'organiser la classe en deux groupes, autour de la réalisation de la tâche "Ecureuil ». Cet extrait met de nouveau en avant la dimension projective et justificative de l'échange : il s'agit de préparer l'activité et d'exposer le bien-fondé des décisions que les enseignants sont en train de prendre. Par rapport aux extraits précédents, ici nous observons que Claudia mobilise une approche expérientielle de la pédagogie spécialisée qui voit dans l'engagement pratique une source d'enrichissement.

Au début de l'extrait, Claudia propose à Jef de réaliser la tâche "Ecureuil » en séchangeant les groupes d'élèves. Jef s'occuperait des élèves en difficulté (les six plus, ligne 4) alors qu'elle prendrait les autres élèves. Jef s'affilie à l'activité de proposition et s'aligne par rapport à ce qui est proposé dans le sens où il permet à Claudia de garder la parole se tenant silencieux. La proposition ayant été faite, Claudia s'engage dans sa justification dans les termes d'une approche expérientielle. En réalisant la tâche avec les élèves en difficulté, Jef pourra se rendre compte de leur manière de se comporter pour les évaluer. L'expérience permettra de confronter l'évaluation de Jef à celle de Claudia, qui est présentée comme ayant tendance à sous-estimer les compétences des élèves. Il s'agit d'un pli que Claudia présente comme lui étant attribué par Jef, mais qu'elle est aussi prête à reconnaître (tu as peut-être raison, ligne 6 ; j'suis d'accord jje souvent j'fais ça j'crois qu'ils sont pas capables et pi en fait ils le sont, lignes 6-8). Jef se montre d'accord avec le portrait que Claudia fait d'elle-même ou, tout du moins, l'invite à poursuivre (ligne 10). Claudia développe alors l'intérêt de sa proposition : le fait de disposer d'une expérience partagée avec les élèves en difficulté constitue la base sur laquelle une 


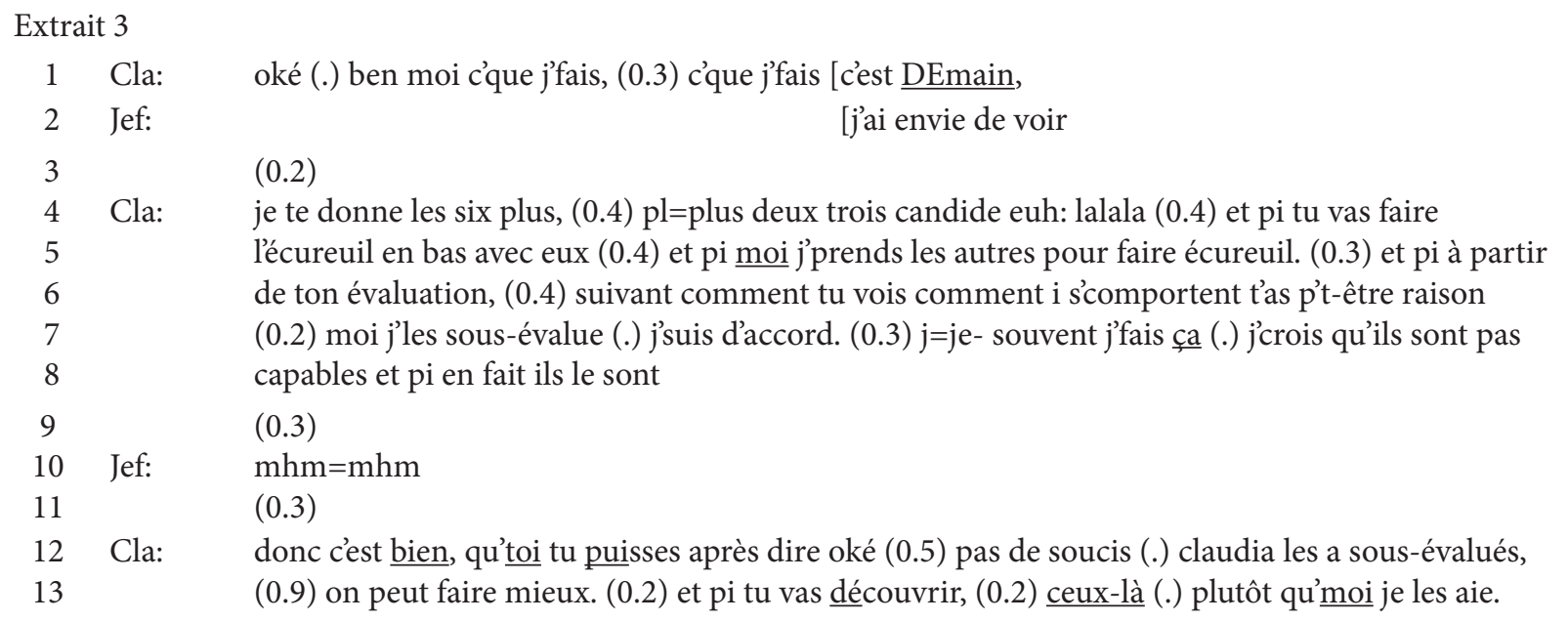

reconsidération des compétences des élèves peut avoir lieu. S'appuyant sur le discours direct, Claudia représente Jef en train de tirer les conclusions de son expérience (oké pas de soucis claudia les a sousévalués on peut faire mieux, lignes 12-13). Avec le pronom on, Claudia laisse entendre que l'expérience pourrait conduire à un changement de pratiques non seulement au niveau de Jef et des élèves, mais également en ce qui la concerne. Toutefois, il est intéressant de signaler que Claudia ne fait que suggérer la possibilité d'un changement de sa propre évaluation, alors qu'elle met en avant surtout la possibilité que Jef s'en démarque et l'utilité de l'expérience pour l’enseignant généraliste (et puis tu vas découvrir ceux-là, ligne 13).

Dans cet extrait, l'intérêt du travail en dyade est souligné par la confrontation dévaluations à l'issue d'expériences communes. L'accord intersubjectif (Rommetveit, 1984) naît d'un dialogue de perspectives, au terme duquel les interactants ajustent leurs significations et parviennent à se comprendre (ou au moins ils en ont l'illusion). Léchange nourrit une ré- flexivité qui consiste à chercher, dans la confrontation avec l'expérience et avec l'autre, des bases pour sa propre conduite.

\section{Un espace d'échange sur l'espace de la classe}

Lextrait 4 met en avant un dernier aspect du travail de projection et de justification des activités éducatives conduit lors de la séance. L'échange permet aux participants de se représenter l'espace physique de la classe et leurs actions à l'intérieur de celui-ci. Il leur permet de partager des projets et des expériences à ce niveau. Jef manifeste son souhait de disposer la classe en forme de $\mathrm{U}$ et indique la manière dont il compte agir au sein de cet espace. Claudia s'aligne en manifestant sa connaissance expérientielle de l'intérêt de la configuration proposée. Pendant l'extrait, les enseignants se déplacent dans l'espace alors qu'ils décrivent les actions à y réaliser.

$\mathrm{Au}$ début d'extrait, Jef exprime son souhait de modifier prochainement la disposition de la classe tout en prenant soin d'apprécier la disposition actuelle (lignes 1-4). Le marqueur phatique tu sais 


\section{Extrait 4}

1 Jef: mais j’ai hâte tu sais, jadore cette disposition avec ces quatre îlots. (0.6) mais j’ai hâte après noël de:

$2 \quad[$ faire ()$=$

3 Cla: [de rechanger

4 Jef: =je veux faire un $u$. jai envie de [faire un u.

5 Cla: [AH oui c'est bien

$6 \quad(0.3)$

$7 \quad \mathrm{mhm}=\mathrm{mhm}(0.5) \mathrm{mhm}=\mathrm{mhm}(0.2)$ c'est [intéressant

[jai envie d'voir ce que c'est.

10 Cla: [ça donne (quoi/ouais),

11 Jef: [ET pi que je puisse me r'trouver [au milieu=

12 Cla:

[c'est tout près-

13 Jef: =d'ce $\mathrm{U}$ et pi tu vois comme ça,

14 Cla: ouais=ouais [ET tu peux aller près=

15 Jef: [jai envie de voir ça, ouais.

16 Cla: =de l'enfant, (0.2) ça=c’est- moi j’aim- j’ai bien aimé (0.5) parc'qu'cest vrai que tu es là:

$17 \quad(1.2)$

18 Cla: et pi tout à coup t’as ton u:: et pi tu vois qu'tu peux entrer dans le u:.

19 Jef: $\quad \mathrm{mhm}=\mathrm{mhm}$

20 Cla: et puis t’approcher vraiement près (0.6) et pi i se voyent moins, i sont comme ça- bon vis-à-vis un

21 p'tit peu:.

22 Jef: Ouais

23 Cla: mais si le u est pas trop=i revient pas trop. un p'tit peu c'est vrai qu'on peut aller proche d'eux:

(ligne 1) appelle à reconnaître l'intérêt de topicaliser la disposition de la classe. Claudia s'aligne avec la proposition d'introduire une disposition en $\mathrm{U}$ (lignes 5 et 7). Jef poursuit en manifestant son souhait d'expérimenter cette disposition (jai envie de voir ce que c'est, lignes 8-9; jai envie de voir ça ouais, ligne 15) et commence à se représenter en train d'agir en son sein. Il se projette et incarne une position où il se retrouve au milieu (ligne 11). Claudia avance un autre trait de la disposition en U, la proximité avec les élèves (lignes 12 et 14). Comme dans l'extrait 2, elle se positionne en tant qu'enseignante experte qui disposerait d'une connaissance de pre- mière main de la disposition que Jef, l'enseignant novice, propose d'introduire. Il est intéressant de remarquer que Claudia fait tout d'abord appel à une tournure au présent pour ensuite opter pour le passé (jaim jai bien aimé, ligne 16) : elle évalue la disposition et justifie son évaluation en évoquant une expérience personnelle aux contours affectifs (jai bien aimé, ligne 16). Elle commence ensuite à se déplacer dans l'espace en même temps quelle décrit les actions à accomplir. Elle utilise le pronom personnel tu qui peut faire référence autant à Jef qu’à un enseignant générique. L'intervention de Claudia combine un partage au sujet de son expérience de la disposi- 
tion avec la projection de l'expérience à venir de Jef. Elle donne à voir les potentialités de la disposition proposée : pour l'enseignant, venir près de l'élève ou s'en éloigner; pour les élèves, être moins en contact entre eux. Jef soutient cette intervention avec son silence et la production de continuateurs (lignes 19 et 22) qu'invitent Claudia à poursuivre. La séance se déroule dans la salle de classe permettant ainsi aux enseignants de se représenter leurs actions à venir dans le même espace où celles-ci auront lieu. L'espace devient donc une ressource sémiotique dans la préparation des activités éducatives et de leur justification. L'extrait montre aussi la dimension expérientielle et incarnée de l'enseignement et l'échange de connaissances entre enseignants. La plus-value de la disposition en U est amenée par Claudia, l'enseignante expérimentée, qui verbalise ses potentialités en termes de proximité et autonomie.

\section{Analyse de l'entretien}

Cette section présente l'analyse de l'entretien entre Claudia, l'enseignante spécialisée, et le chercheur (Che), réalisé après l'examen de la séance entre enseignants. L'entretien confirme que Claudia considère l'activité enseignante à partir d'une ap- proche expérientielle, une notion qui avait émergé de l'analyse de la séance. L'extrait 5 confirme aussi que Claudia propose à Jef d'adopter cette approche et l'invite à faire les expériences quelle-même a eues. Selon elle, la confrontation directe avec les difficultés des élèves amène une plus-value dans les compétences de l’enseignant généraliste.

La confrontation à la résistance des élèves en difficulté est avancée par Claudia comme la possibilité pour Jef de prendre de la distance par rapport à ses certitudes d'enseignant généraliste. Cette confrontation est vue comme une possibilité d'enrichissement pour Jef en tant que professionnel. Pour Claudia, elle amène aussi un questionnement qui sollicite la créativité, qui bénéficie donc de l'expérience et est utile pour répondre aux défis que pose celle-ci. La confrontation de Jef aux résistances des élèves est ensuite réinvestie dans le rapport dyadique entre les deux enseignants : ils partagent une expérience commune que Claudia considère comme une possibilité d'accord intersubjectif (on se crée une culture commune quand je dis difficulté le mot difficulté il recouvre une même réalité, lignes 16-17). L’entretien permet également de confirmer une autre dimension de la séance mise en avant par l’analyse de

\section{Extrait 5}
Che: là je vais parler par rapport à ce thème qui est sorti de ton interaction avec Jef que je trouve intéressant donc en fait à un moment tu fais aussi mention d’expérimenter en fait d’apprendre par lexpérience est-ce que tu peux men dire un peu plus par rapport à ça dans le cas en fait c'est Jef qui tu lui dis ben prends les élèves à moi ( ) de $6+$ donc effectivement je pense alors là c'est donc pour lui c'est en tant qu'enseignant c'est en tant que
professionnel je pense que lui euh d'expérimenter de l'intérieur quelles sont les comment est-ce
Cla: quon travaille avec les élèves de $6+$ va augmenter ses compétences (...)
loccasion de se confronter à du questionnement a de la résistance à des difficultés$$
\text { (...) }
$$
plus tu fais d'expériences qui sont les tiennes plus tu peux ensuite avoir dans ta tête enfin être créatif la créativité elle se construit elle naît de l'expérience et puis l'expérience crée la créativité
Cla: (...)
fais l’expérience dêtre avec un groupe délèves en difficulté ou fais l’expérience d’organiser une tâche pour eux spécifiquement pour eux puis si ça marche pas oké tant pis mais au moins ceest
Cla: à travers c'est sur la base de ça qu’on se crée une culture commune quand je dis difficulté le mot difficulté il recouvre une même réalité 


\section{Extrait 6}

1 Cla: je dis à Jef non mais c'est trop difficile le test il faut aménager ça ça ça et pis lui il soppose à moi

$2 \quad$ puis il dit non je crois qu’on peut essayer de compter sur leurs compétences

3 (..)

$4 \quad$ il me retire du côté même des compétences que javais même plus évaluées sur ces élèves-là et

5 Cla: souvent les élèves bénéficient de du fait qu'il ait moins peur que moi il est moins lié à la difficulté

$6 \quad(\ldots)$

7 c'est ça quà un moment euh c'est aussi euh tu évoques tu tu sous-évalues euh tu as cette cette

8 tendance tu tu tu dis euh de sous-évaluer tes élèves donc c'est un peu justement ce que tu disais

9 là

son enregistrement : dans l'extrait 6, Claudia présente la séance comme une opportunité de confronter les évaluations que les enseignants font des compétences des élèves et de les modifier dans leur bénéfice.

Lors de la séance, il arrive que les enseignants soient en désaccord au sujet des compétences des élèves et des tâches appropriées à celles-ci. Pour Claudia, les enfants bénéficient de cette confrontation dopinions au moment de la préparation des activités éducatives. Claudia considère qu'elle sousévalue parfois ses élèves du fait dêtre plongée dans le milieu de la pédagogie spécialisée. Jef lui permet de renouer avec les attentes ordinaires parce qu'il est moins touché par la difficulté dans sa profession en tant qu'enseignant généraliste. Claudia pense que les enfants bénéficient de son apport qui permet de contrebalancer la perspective professionnelle de Claudia. La confrontation offre à Claudia la possibilité de remettre en question les tâches quotidiennes qu'elle prodigue aux enfants et des pratiques d'amé- nagement des tâches pour les enfants en difficulté, telles que la réduction du nombre des questions ou l'aménagement des échelles de notation. L'entretien confirme l'importance de répondre au défi des classes inclusives : trouver un équilibre entre proposer des tâches stimulantes pour les élèves et tenir compte de leurs difficultés.

Dans l'extrait 7, Claudia explicite la manière de répondre à ce défi : travailler sur les tâches.

Claudia considère l'accord entre enseignants comme une clé à la bonne marche des classes inclusives. Cet accord se construit lors des séances, mais bénéficie également du suivi en commun de formations spécialisées. Celles-ci amènent les enseignants à de nouvelles réalisations : on sest rendu compte qu'il faut beaucoup plus travailler sur les tâches (lignes 6-7). Claudia fait aussi référence à différentes manières de sengager dans des activités communes : être dans la spontanéité; se coordonner pour des aspects purement organisationnels ; s'engager également dans une réflexion sur la tâche. Pour

\section{Extrait 7}

1 Che: et puis comment tu généralement dans l'organisation des tâches tu trouves cet équilibre parce que

$2 \quad$ justement il y a il y a un peu cette idée aussi qui en ressort euh de euh de défavoriser les bons et

3 puis en même temps on défa on défavoriserait aussi les les moins bons

$4 \quad(\ldots)$

5 on est d'accord et Jef et moi parce qu'on est allé en formation une matinée avec des gens qui

6 réfléchissent au co-enseignement donc on s'est rendu compte qu'il faut beaucoup plus travailler

$7 \quad$ Cla: sur les tâches

$8 \quad(\ldots)$

9 parfois on est dans la spontanéité puis on se coordonne comme ça mais on perd en qualité on

10 perd en qualité et on sait que c'est ça et c'est là que c'est le maillon faible 
Extrait 8

1 Cla: c'est vrai que la tâche il faudrait presque la la la la penser en trois pour trois groupes

$2 \quad(\ldots)$

3 comment on fait une même tâche comment pour le groupe des bons on va faire la tâche

4 Cla: comment on va faire la tâche pour le groupe des faibles et comment on va faire la tâche pour

5 ceux qui roulent

$6 \quad(.$.

7 est-ce que sur cette tâche il existe des prolongements pédagogiques si oui lesquels

$8 \quad(\ldots)$

9 Cla: pour les faibles ben on peut par exemple décider oké là la tâche elle est très complexe donc on va

10 donner le matériel ou bien la tâche est un peu difficile on va la raccourcir

$11 \quad(\ldots)$

12 Cla: les très bons élèves ils ont un projet personnalisé donc de temps en temps on leur dit vous allez

13 sur le projet personnalisé et puis là ils doivent aller sur l'ordinateur faire une petite recherche

14 d'informations

Claudia, il est dommage de consacrer la séance uniquement à discuter des aspects dorganisation pratique alors qu'une réflexion plus poussée au niveau pédagogique serait souhaitable. L'entretien permet à Claudia d'expliciter la nature du travail qu'elle souhaiterait réaliser lors des séances.

Lextrait 8 met en évidence ce quen quoi consisterait une réflexion sur la tâche répondant aux défis de la classe inclusive.

Claudia pense les tâches dans une perspective triadique : les bons élèves; les élèves moyens ; les élèves en difficulté d'apprentissage, dont elle se charge. Elle propose de décliner chaque tâche de trois manières différentes afin de mobiliser le meilleur de chacun. Avec les élèves sans difficultés, il y a souvent la possibilité de mettre en place des prolongements pédagogiques offerts dans les manuels ou que les enseignants peuvent créer afin de permettre un équilibre entre les compétences effectives de l'enfant et ce que l'on attend de lui. Claudia s'exprime sur comment traiter la difficulté : avec Jef, elle s'active à individualiser les tâches. Lorsqu'il s'agit d'activités complexes avec des élèves en difficulté, ils peuvent décider de l'aménager ou de mettre à disposition de l'élève des ressources complémentaires. Lorsqu'il s'agit d'élèves ayant de l'aisance à l'école, les ensei- gnements font appel à des projets personnels qui incitent le développement exploratoire de l'enfant.

\section{Discussion et conclusion}

Notre étude montre que la séance entre enseignant spécialisé et enseignant généraliste en milieu inclusif est un espace déchange aux multiples fonctions. Elle permet de préparer les activités éducatives en confrontant les propositions des deux enseignants. Ceux-ci se projettent dans la réalisation des activités et mettent en scène les dialogues qu'ils pourront avoir avec les élèves ainsi que les actions à entreprendre. Il s'agit d'une représentation qui a une dimension langagière mais également spatiale puisque les enseignants testent également des déplacements et de dispositions in absentia des élèves. En avançant leurs propositions, les enseignants sont invités à les justifier, c'est-à-dire à exposer les éléments qui justifieraient leurs choix pédagogiques. La séance est aussi un espace de socialisation où les enseignants endossent leurs rôles respectifs, apprennent à connaître celui de l'autre partie et sont invités à se mettre à la place de l'enseignant partenaire. Tout en mettant en évidence de différentiels de connaissance et expérience, la séance permet aux enseignants de les combler en mettant au bénéfice 
de l'enseignant novice l'expertise de sa collègue. La séance est surtout un moment de confrontation des évaluations des élèves par les enseignants et de réflexion sur les tâches les plus appropriées à cellesci. Les élèves bénéficient d'un échange qui peut permettre aux enseignants de dépasser des évaluations qui sur- ou sous-estimaient leurs compétences. Ils bénéficient aussi de solutions créatives que léchange permet de faire émerger, pour dépasser les difficultés de l'enseignement en classe inclusive.

Lanalyse de la séance montre que l'échange conduit les enseignants à s'engager dans une réflexion au sujet de leurs activités éducatives. Il s’agit d'une réflexivité commune qui prend forme de manière imbriquée à léchange, au fur et à mesure que les participants enchaînent leurs tours de parole. Les significations "ne sont repérables ni dans la prise de parole de l'un, ni dans la prise de parole de l'autre (...) Il y a une efficacité du dialogue qui fait que ce qui se produit dépasse ce que chaque locuteur aurait pu produire séparément" (Salazar Orvig, 1999, p. 163). Ce phénomène peut se rapprocher de la posture de Schön (1994) concernant la réflexion dans l'action comme capacité d'autorégulation qui s'active lors d'interactions sociales. Ce phénomène renvoie également à l'importance pour les participants de rendre visible la rationalité de leur conduite (Arcidiacono, 2015). Lanalyse de la séance manifeste l'importance pour les enseignants en classe inclusive d'un alignement réciproque : le risque de rupture est paré à coup de justification des propositions, dévocation de connaissances et d'expériences permettant de les avancer. Les enseignants font preuve de réflexivité dans l'action et sur l'action (Vacher, 2011), de manière imbriquée : l'attribution d'un discours à tenir à l'avenir ou la représentation de déplacements dans l'espace à effectuer une fois en classe témoignent de pratiques réflexives qui ont lieu dans l'action, lors de la séance, mais qui visent une réflexion sur laction éducative, en contact avec les élèves. En définitive, la séance stimule la capacité de mise à distance, chez les enseignants, envers la pratique effective afin de réfléchir le faire (Vacher, 2011).

Cette étude a dégagé un ensemble de réflexions qui nourrissent le débat à propos de la pratique d'enseignement en milieu inclusif. Il s'agit de penser la dimension fortement interactionnelle du cadre didactique : la manière de façonner les dynamiques de coopération au sein de la dyade enseignant spécialisé-enseignant généraliste, de manière à travailler dans le bénéfice des élèves. Dans le but de prolonger la réflexion, une analyse comparative sur d'autres processus réflexifs pourrait permettre de rendre compte d'approches plus interventionnistes, allant au-delà de léchange lors de séances entre enseignants. Elle pourrait aussi apporter des éclaircissements ultérieurs sur des dimensions psychosociologiques liées aux pratiques éducatives d'enseignants généralistes et spécialisés.

\section{Références}

- Arcidiacono, F. (2013). Intersubjectivité et agency dans la conversation quotidienne: pratiques de socialisation en contexte. In C. Moro, N. Muller Mirza \& P. Roman (Eds.), L'intersubjectivité en questions. Agrégat ou nouveau concept fédérateur pour la psychologie? (pp. 292-312). Lausanne: Antipodes.

- Arcidiacono, F. (2015). Argumentation and reflexivity. In G. Marsico, R. Andrisano-Ruggieri \& S. Salvatore (Eds.), The Yearbook of Idiographic Science. Volume 6: Reflexivity and Change in Psychology (pp. 169-193). Charlotte: Information Age Publishing.

- Arcidiacono, F., Baucal, A., \& Buđevac, N. (2011). Doing qualitative research: The analysis of talk-in-interaction. In A. Baucal, F. Arcidiacono \& N. Buđevac (Eds.), Studying interaction in different contexts: A qualitative view (pp. 17-45). Belgrade: Institute of Psychology. 
- CDIP (2017). Accord intercantonal sur la collaboration dans le domaine de la pédagogie spécialisée. Berne: Conférence suisse des directeurs cantonaux de l'instruction publique.

- Coffey, A., \& Atkinson, P. (1996). Making sense of qualitative data. Thousand Oaks: Sage.

- Czyzewski, M. (1994). Reflexivity of actors versus reflexivity of accounts. Theory, Culture and Society, 11, 161-168.

- Dos Santos Mamed, M. (2014). Je suis, tu es, il/elle est: un regard psychosocial sur une classe de français langue étrangère. Mémoire, Faculté des sciences sociales et politiques, Université de Lausanne.

- Grossen, M., \& Salazar Orvig, A. (2011). Third parties' voices in a therapeutic interview. Text \& Talk, 1(31), 54-76.

- Houssaye, J. (1992). Le triangle didactique: théorie et pratiques de l'éducation scolaire. Berne: Lang.

- Jefferson, G. (2004). Glossary of transcript symbols with an introduction. In G. H. Lerner (Ed.), Conversation Analysis: Studies from the first generation (pp. 13-31). Philadelphia: Benjamins.

- Krummheuer, G. (2000). Studies of argumentation in primary mathematics education. Zentralblatt für Didaktik der Mathematik, 32(5), 155-161.

- Lynch, M. (2000). Against reflexivity as an academic virtue and source of privileged knowledge. Theory, Culture and Society, 17(3), 26-54.

- Mondada, L., \& Pekarek Döhler, S. (2000). Interaction sociale et cognition située. Quels modèles pour la recherche sur l'acquisition des langues? Acquisition et interaction en langue étrangère, 12, 1-21.

- Mondada, L., \& Pekarek Döhler, S. (2006). Compétences et pratiques sociales. Etudes critiques. Bulletin suisse de linguistique appliquée, 84, 1-8.

- Pontecorvo, C., \& Arcidiacono, F. (2007). Famiglie allitaliana. Parlare a tavola. Milan: Cortina.

- Pontecorvo, C., \& Arcidiacono, F. (2014). Social interactions in families and schools as contexts for the development of spaces of thinking. In T. Zittoun \& A. Iannaccone (Eds.), Activity of thinking in social spaces (pp. 83-97). New York: Nova Science Publishers.

- Radišić, J., \& Baucal, A. (2016). Using video-stimulate recall to understand teachers' perceptions of teaching and learning in the classroom setting. Psihološka istraživanja, 19(2), 165-183.

- Roger, J.-L., Ruelland, D., \& Clot, Y. (2007). De l’action à la transformation du métier: lactivité enseignante au quotidien. Education et sociétés, 19(1), 133-146.

- Rommetveit, R. (1984). The role of language in the creation and transmission of social representations. In R. M. Farr \& S. Moscovici (Eds.), Social Representations (pp. 331-359). Cambridge: Cambridge University Press.

- Sacks, H., Schegloff, E. A., \& Jefferson, G. (1974). A simplest systematics for the organization of turn-taking for conversation. Language, 50, 696-735.

- Salazar Orvig, A. (1999). Les mouvements du discours. Paris: L'Harmattan.

- Schön, D.-A. (1994). Le praticien réflexif. A la recherche du savoir caché dans l’agir professionnel. Montréal: Editions logiques.

- Vacher, Y. (2011). La pratique réflexive. Un concept et des mises en œuvre à définir. Recherche \& Formation, $66,65-78$. 


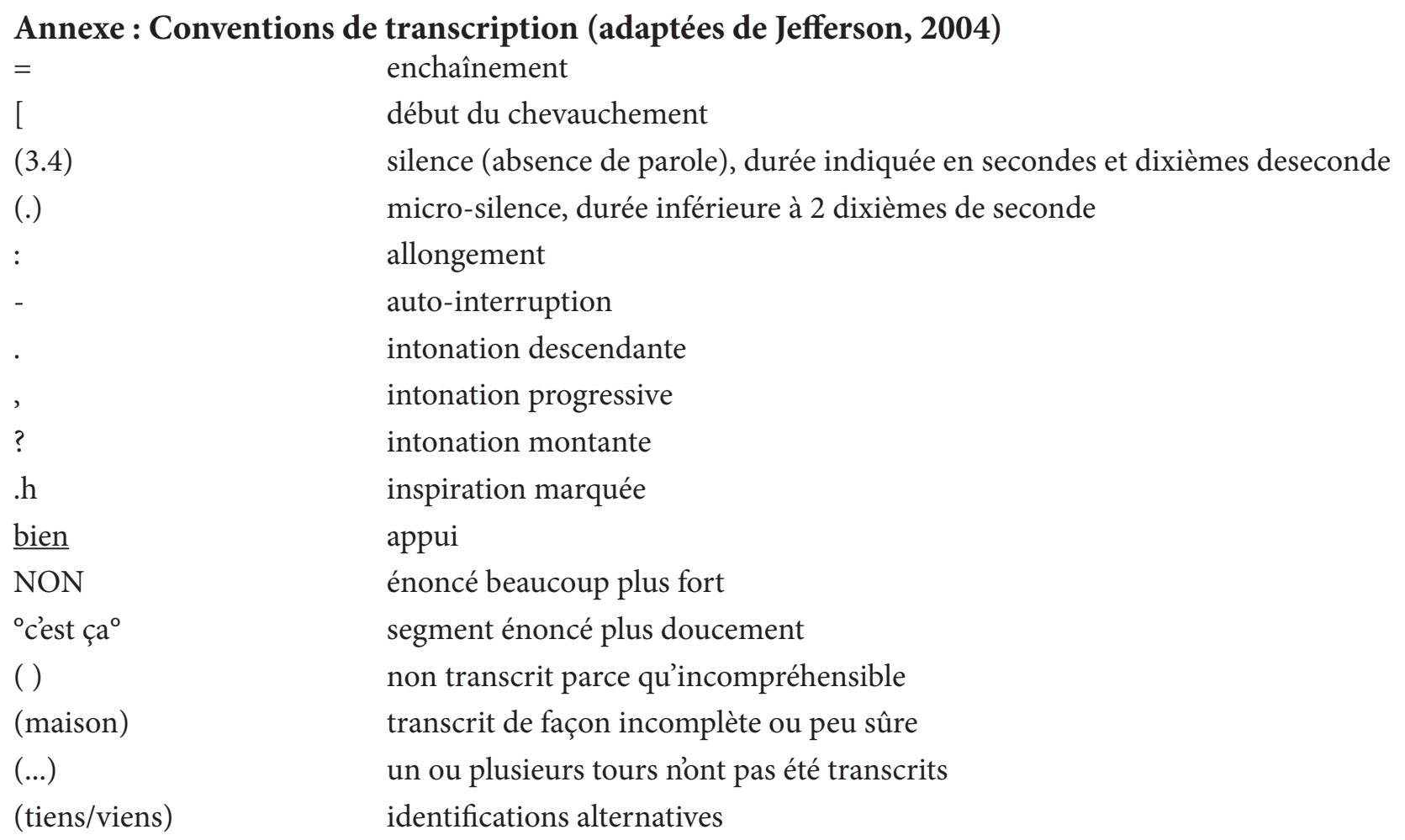




\section{Франческо Арчидијаконо \\ Александар Безенсон \\ Естер Гонзалес-Мартинез}

\section{РАДНИ САСТАНЦИ НАСТАВНИКА ИНКЛУЗИВНЕ НАСТАВЕ: ПРОСТОР ЗА РАЗМЕНУ ИДЕЈА}

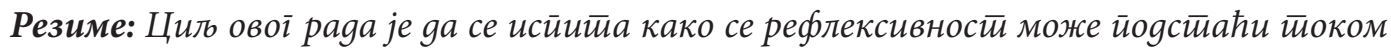

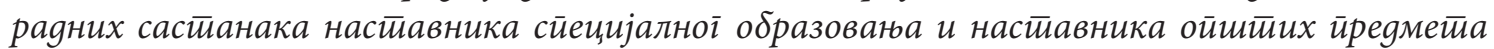
који сарађују у оквиру инклузивне школе. Инклузивни кониекксй је ирростиор инииетрације

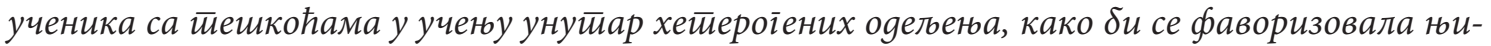
хова ауйономија, ирроцес сочијализације, стичиане зната и иромовисала оивворености ире-

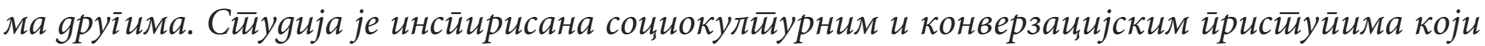

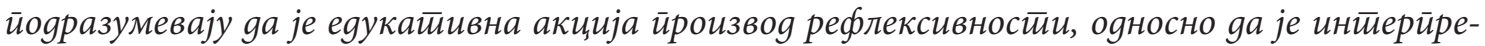

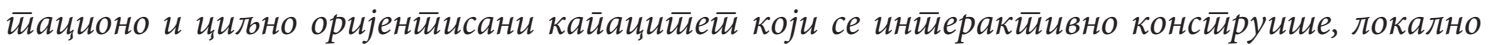
је тоциран и доїоворен ииоком дискурзивних размена између особа које су у инитеракцији.

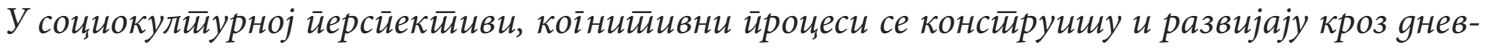

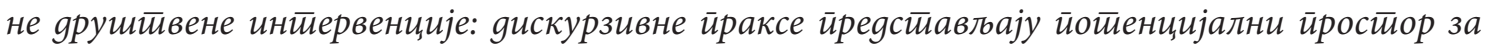
размену йоком које учесници зајеgнички йроизвоgе gискурс и ирреіоварају о йреgметиу gис-

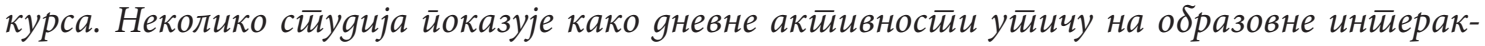
иије, честио ирретииостиављајући аріументиатиивну gимензију која gовоgи у йитиане валия-

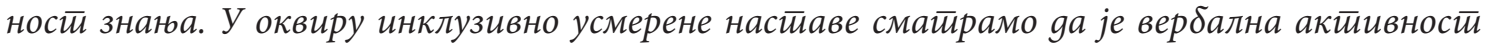

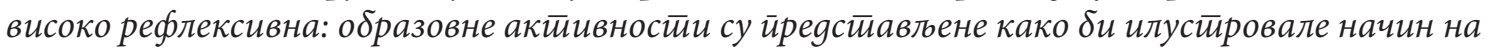
који настиавници йоказују рефлексивности и како она gовоgи gо йеgаїошкоі йозиционираға йоком друшишвених иниееракиија. Фокусиране на раяне сесије омоіућава боле разумевате

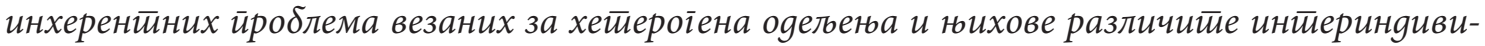
gуалне ресурсе. Такође, ова врсииа фокуса йомаже gа разумемо како насиианици креирају

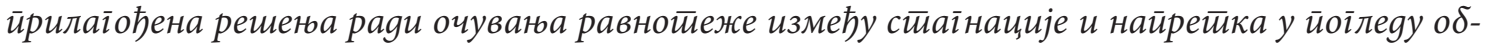
разовних избора. Динамика у раяу између настиавника оититихх ирреgметиа и настиавника сиее-

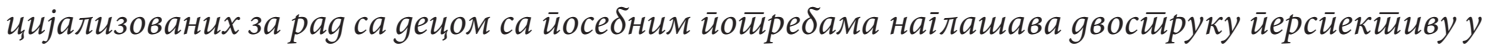

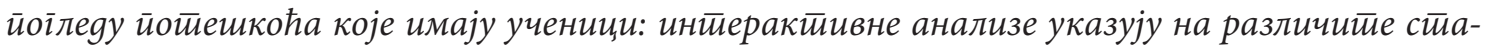

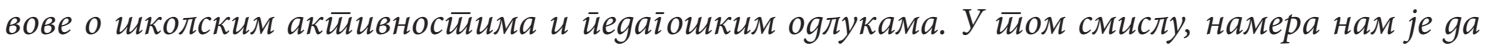

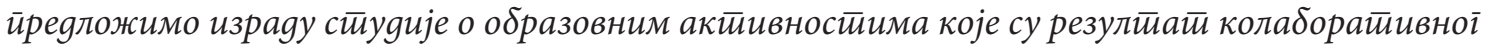
раgа, креиране зајеgничким найорима кроз реблексивносй у акцији. Раgи болееі разумевать значајних йитиаюа која се јавтьају у инклузивној настияви, као и йојаве рефлексивностии, насйавниии су обезбеgили ауgио-снимке са раяних састианака и ирихватиили да учестивују у инgивияуалним иниетевјуима са истираживачима.

Извршена је анализа разі̄овора на раяним састианиима како би се објаснила йроизвоgюа рефблексивности на лииу месииа. Налази йоказују gа се кроз вербалне размене насииа-

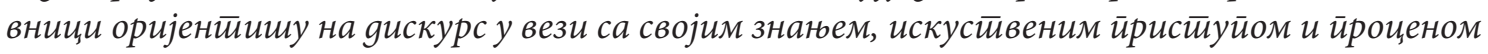




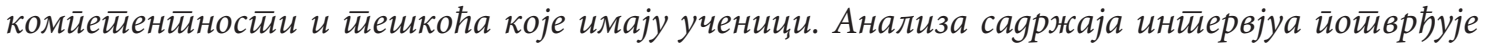
ове резулитайе и омоіуућава насииавницима gа објасне своје оgїоворе који се односе на инклу-

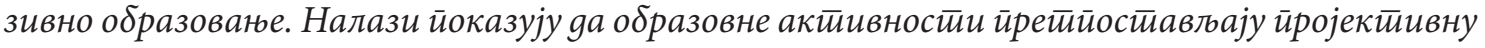

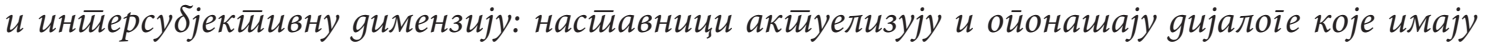
са стиудениичма, као и сойсиввене йосйуйке. Језик функиионише у синеріији са заузетиим йо-

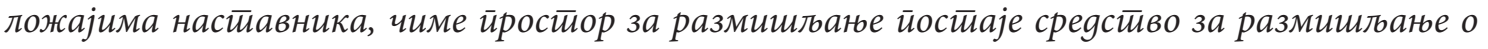

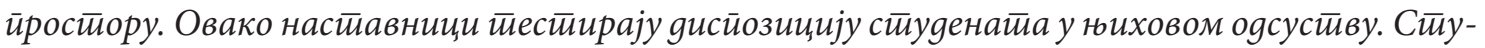

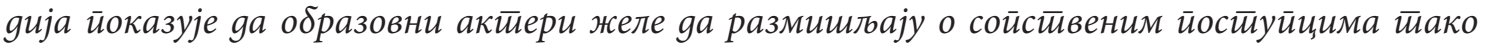
шимо своје йонашаюе чине вияльвим и рационалним. Иако настиввници јавно йоказују своја

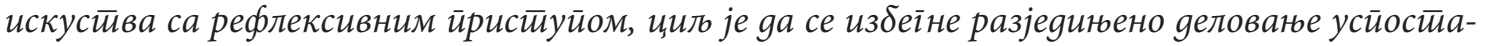

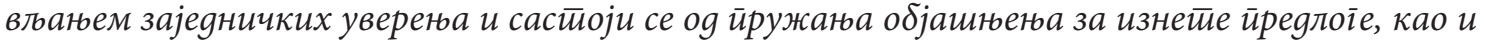
узајамноі йодржаваюа кроз дельнье искусииава и знаюа. Овако ефикасно йланирана размена

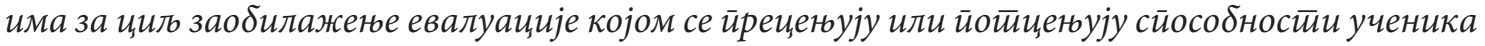

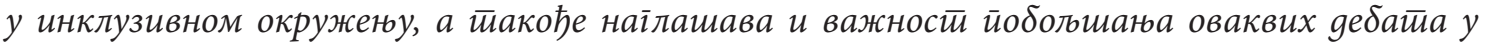
буgућим истираживаюима у образоваюу.

Кључне речи: настиавник, инклузивна настиава, размена мишљеюа насйавника, рефлексивност. 